\title{
FINE STRUCTURE AND SYNAPTOLOGY OF THE NITRERGIC NEURONS IN MEDIAL SEPTUM OF THE RAT BRAIN
}

\author{
Katalin Halasy, * Balázs SzŐKe and Gergely Janzsó \\ Department of Anatomy and Histology, University of Veterinary Sciences, \\ H-1078 Budapest, István u. 2. Hungary
}

(Received: June 14, 2016; accepted: September 6, 2016)

\begin{abstract}
The nitrergic neuron population and certain aspects of their connectivity (peptidergic inputs, co-localization with GABA, synaptic target distribution) were studied in the medial septum of the rat brain. The histochemical localization of NADPH diaphorase and immunohistochemical identification of nNOS at light and electron microscopic level was applied. Double-labeling experiments with galanin and leucine enkephalin, moreover the postembedding GABA immunogold staining was also carried out. NADPH diaphorase- and nNOS-immunopositive neurons could be identified inside the borders of medial septum. Out of their peptidergic inputs galanin- and leucine enkephaline-immunopositive varicose fibers were found in close apposition with nNOS-immunopositive neurons. Based on fine structural characteristics (large indented nucleus, thin cytoplasmic rim, lack of axosomatic synapses) the nitrergic neurons are suggested to be identical with the septal cholinergic nerve cells. Their boutons established asymmetrical synapses mainly on dendritic shafts and spines, some of which were also nNOS-immunopositive. A lower amount of nNOS-immunopositive boutons of presumably extrinsic origin were found to be GABAergic.
\end{abstract}

Keywords: nNOS - NADPH - neuropeptides - GABA-synaptology

\section{INTRODUCTION}

The septal area, as an interface, connects the limbic telencephalic regions with hypothalamic and brainstem areas. A major anatomical component of the rat septum is the medial septum/diagonal band of Broca (MS/DB) complex. The MS/DB contains primarily cholinergic $[32,35]$ and GABAergic projection neurons, which are believed to play a pivotal role in the generation of theta frequency oscillation through the wellknown septo-hippocampal interconnection [11, 12, 25]. The MS/DB relays motivational, emotional and autonomic information to the cortical areas via peptidergic afferents from the hypothalamus and monoaminergic afferents from the midbrain [23]. Cholinergic neurons within this complex are considered to be the primary source of acetylcholine to the cortex and hippocampus. However, the MS/DB complex also contains GABAergic [27] and glutamatergic [39] neurons, and more than the half of the neurons within the MS/DB were determined to be non-cholinergic [2,

*Corresponding author; e-mail address: halasy.katalin@univet.hu 
$3,6,36]$. The focus of research on cholinergic neurons within this complex aims to understand their role in etiology of certain dementias [8, 22]. The septohippocampal pathway is important for learning and memory-related behaviors, and degeneration of cholinergic MS/DB neurons can be diagnosed in neuropathological disorders such as Alzheimer's disease [4, 20, 41].

Nitric-oxide (NO) is a membrane-permeable signal molecule, synthesized in neurons by a constitutive isoenzyme called neuronal nitric-oxide synthase (nNOS). It has a wide range of modulatory effects on neuronal transmission by cGMP-dependent mechanisms [14]. Actions of nNOS have been associated with control of sleep, appetite, thermoregulation $[16,30]$ neural development $[15]$ and synaptic plasticity $[17$, 19]. Depending on the concentration of NO, glutamate release is either enhanced or decreased, and the glutamate stimulates the NMDA and AMPA/kainate receptors located on cholinergic neurons $[34,38]$. Similar, concentration-dependent direct effects were measured on the GABA release [18] and an indirect effect on $\mathrm{GABA}_{\mathrm{A}}$ receptors of cholinergic cells [33]. Thus, the acetylcholine release is modulated indirectly in a biphasic manner depending on NO concentrations.

The aim of our investigation was primarily to study the ultrastructure and synaptology of the nitrergic elements in the MS/DB area with correlated light and electron microscopic immunohistochemical studies. Their connection with the extensive local peptidergic system, distribution and excitatory/inhibitory character of their postsynaptic targets were also studied.

\section{MATERIALS AND METHODS}

The experiments were performed on 6 young adult male Wistar rats of the same age weighing 230-250 g. All animal procedures were conducted in accordance with the guidelines set forth by the European Communities Council Directive of 63-2010 and the Animal Health and Welfare Institute of the Veterinary University, and all efforts were made to keep animal stress, suffering and discomfort to a minimum level. The Local Animal Welfare Association permitted and controlled the experiment.

\section{Fixation and tissue preparation}

The animals were anaesthesized with an intraperitoneal injection of Nembutal (Na-pentobarbital $100 \mu \mathrm{l} / 250 \mathrm{~g}$ body weight) then transcardially perfused with heparinized saline for $5 \mathrm{~min}$, followed by a fixative containing $4 \%$ paraformaldehyde, $0.1 \%$ glutaraldehyde in $0.1 \mathrm{M}$ phosphate buffer $(\mathrm{PB})(\mathrm{pH} 7.4)$ for $30 \mathrm{~min}$. Brains were removed and postfixed overnight in the same, but glutaraldehyde-free fixative. Sixty $\mu \mathrm{m}$ thick coronal vibratome sections were cut from the septal area (from bregma 1.70 $\mathrm{mm}$ to $-0.40 \mathrm{~mm}$ ). 


\section{Pre-embedding immunocytochemistry}

nNOS-immunocytochemistry was applied using the free-floating pre-embedding

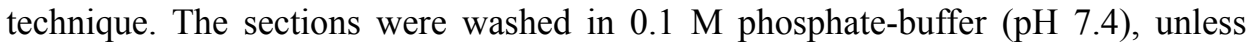
otherwise stated. For light microscopy each solution contained $0.4 \%$ Triton X-100 in order to increase the penetration of the antibodies, whereas sections for ultrastructural studies were treated in solutions without Triton X-100.

Sections were kept in 10, 20 and $30 \%$ sucrose in $0.1 \mathrm{M}$ PB successively, then freeze-thawed in liquid nitrogen to increase the penetration of the antibodies. This was followed by three washes, and treatment with $1 \%$ hydrogen peroxide for 30 minutes to block endogenous peroxidase activity, then $10 \%$ normal goat serum was applied for the blocking of non-specific binding sites for 45 minutes at room temperature. After a short rinse, sections were incubated overnight with the primary monoclonal mouse anti-NOS1 antibody (Santa Cruz Biotechnology, SC-5302) in 1:500 dilution overnight at $4{ }^{\circ} \mathrm{C}$ with continuous shaking. Following several rinses, biotinylated anti-mouse immunoglobulin (Vector Laboratories, 1:100) was used as a secondary antibody for 4 hours at room temperature. This was followed by several rinses and incubation in avidin-biotin-horseradish peroxidase (ABC Elite Kit, Vector Laboratories, 1:100) for 2 hours, then the sections were washed three times in $0.05 \mathrm{M}$ TRIS buffer ( $\mathrm{pH}$ 7.6). The immunoreactive structural elements were visualised with 3,3'-diaminobenzidine (DAB).

The sections for light microscopy were mounted onto gelatine-coated slides, dehydrated in a series of ethanol, cleared in xylene and mounted in DePeX.

\section{Light microscopic double-labeling experiments}

Double-labeling experiments were done in order to reveal possible contacts between nNOS-positive cells and peptidergic axon terminals. Incubation with the primary antibodies (mixtures of nNOS - leu-enkephalin and nNOS - galanin, resp.) was applied in one step and first the peptidergic axons were visualized. The used primary antibodies were rabbit anti-galanin (Serotec, PEPA31, 1:500), rabbit anti-leuenkephalin (Sigma. L 8516, 1:500) combined with monoclonal mouse anti-nNOS as described above. The secondary antibody for the polyclonal primary antisera was biotinylated goat-antirabbit (Vector Laboratories, 1:100) followed by ABC (see above) and for the visualization of the peptidergic elements the DAB-nickel method resulting in dark blue-to-black staining was applied. This was followed by the visualization of nNOS-immunopositive cells as described above and the simple DAB reaction staining immunopositive elements in brown colour was applied to distinguish between the two immunoreactions. 


\section{Electron microscopy}

Sections for electron microscopy were postfixed in $1 \%$ osmium tetroxide in $0.1 \mathrm{M} \mathrm{PB}$ for 35 minutes and contrasted with $70 \%$ ethanol saturated with uranyl acetate. After complete dehydration in ascending ethanol series and propylene oxide, sections were mounted on slides and flat-embedded in Durcupan (Fluka) resin. After polymerization at $56{ }^{\circ} \mathrm{C}$ the sections were viewed under a light microscope, areas of MS rich in nNOS-immunopositive elements were selected and re-embedded for ultrathin sectioning. Sixty $\mu \mathrm{m}$ ultrathin serial sections were cut, mounted on single-slot copper grids and examined in electron microscope.

Control sections were incubated in the same way except the primary antibody was omitted from the buffer. Such sections did not exhibit any immunostained structural elements.

\section{Postembedding immunogold staining}

Ultrathin sections containing pre-embedding labeling for nNOS were mounted on nickel grids for postembedding GABA labeling. The procedure was performed on droplets of reagents and solutions in a humidified Petri dish. In order to reveal binding sites free from resin and osmium-tetroxide, $1 \%$ periodic acid was used for $10 \mathrm{~min}$ utes, followed by $1 \%$ sodium-periodate for 10 minutes. One percent ovalbumine droplets were used for 30 minutes as blocking solution, and GABA 9 [21] was the primary antiserum in 1:1000 and 1:2000 dilution at $4{ }^{\circ} \mathrm{C}$ for overnight. Goat antirabbit immunoglobulin decorated with gold particles (Gold Gar IgG, 10 and $15 \mathrm{~nm}$, Aurion, in 1:10 and 1:20 dilution) was used as secondary antibody for 2 hours. Washing solutions between each steps were distilled water, TRIS-buffered saline (TBS), and Gold-GAR buffer (1\% BSA and $0.5 \%$ Tween 20 in 0.05 M TRIS buffer, $\mathrm{pH}=7.4$ ), respectively. We used saturated uranyl-acetate and lead-citrate as contrasting reagents. Electron micrographs were prepared in a JEOL 100C EM equipped with a digital camera and connected with a computer using AnalySIS program.

\section{NADPH-diaphorase histochemistry}

For NADPH-diaphorase histochemistry animals were anaesthetized-perfused as described previously, except the fixative was $4 \%$ paraformaldehyde in $0.1 \mathrm{M}$ PB $(\mathrm{pH}=7.4)$ only. Sixty $\mu \mathrm{m}$ vibratome sections were incubated in $0.2 \%$ Triton X-100 in $0.1 \mathrm{M} \mathrm{PB}$ for 12 hours at $4{ }^{\circ} \mathrm{C}$, then transferred into an incubation solution containing: $0.1 \mathrm{M}$ PB $(\mathrm{pH}=8.00), 0.8 \%$ TritonX-100, $1 \mathrm{mM}$ NADPH, $0.8 \mathrm{mM}$ NBT (Nitroblue Tetrazolium) for 2 hours. After the incubation sections were washed in PB and mounted as described previously. Light micrographs were viewed and photographed in an Olympus light microscopy. 


\section{RESULTS}

\section{Distribution of nNOS-immunopositive and NADPH-diaphorase positive neuronal elements in the medial septum}

Both immunocytochemistry and histochemistry revealed comparably stained neuronal elements in the studied brain area (Fig. 1). In the medial septum the majority of both immunopositive (Fig. 1A) and histochemically detectable (Fig. 1B) neurons was located laterally from the midline, at the border of lateral and medial septal areas. The stained neurons were small, oval or rounded, their diameter varied between 15-25 $\mu \mathrm{m}$ (Fig. 1C, D). Their nuclei were large, round or ovoid. The proximal segments of their 2-3 dendrites were also stained with both methods. The punctate staining in the histochemical sections refers to a large number of synaptic terminals. Immunopositive varicose axons also appeared in the immunocytochemically stained sections.
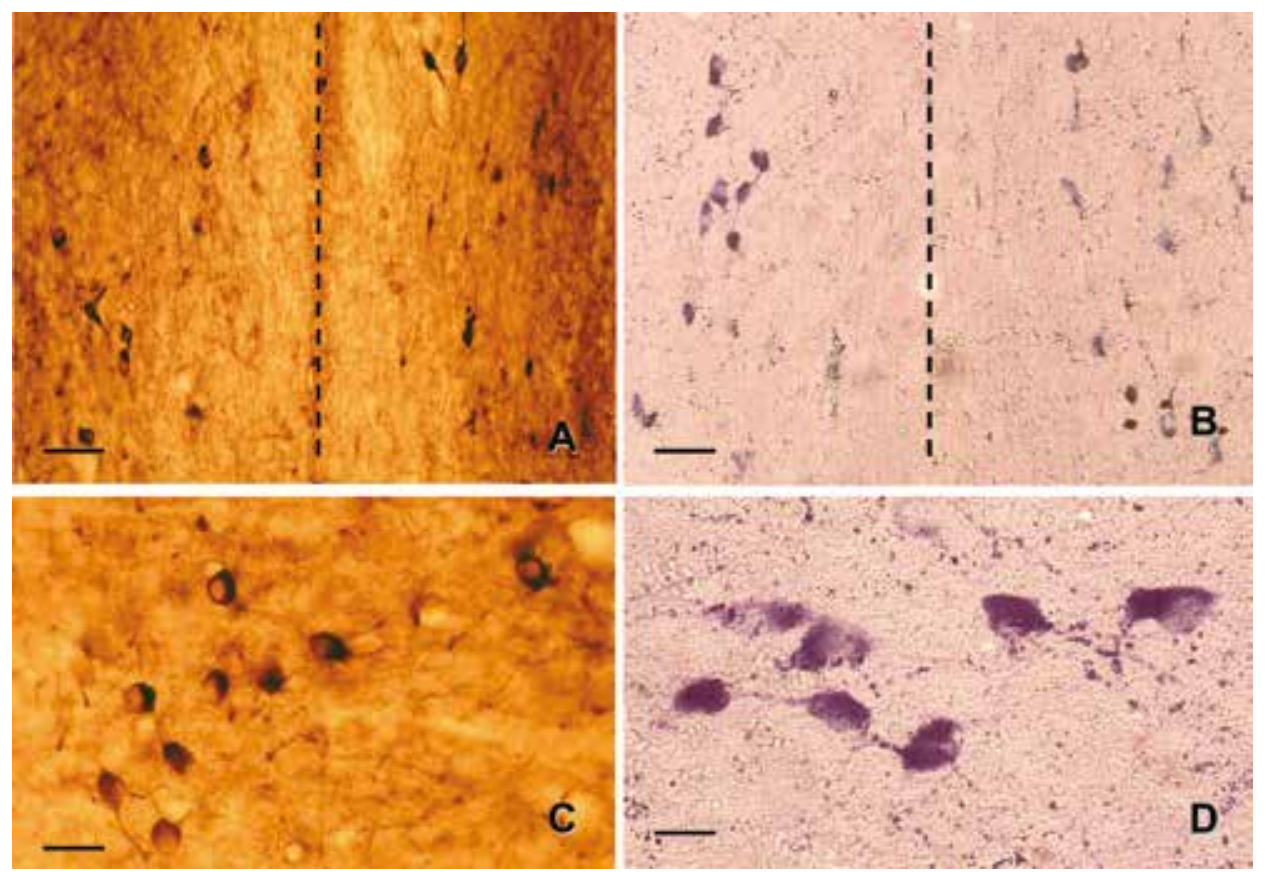

Fig. 1. Comparison of staining patterns with nNOS-immunocytochemistry (A, C) and NADPHdiaphorase enzyme histochemistry (B, D). A: nNOS-immunocytochemistry revealed small neurons with round nuclei laterally from the midportion (dashed lines) of MS. B: Cells with similar size, morphology and distribution were stained with the NADPH-diaphorase enzyme histochemistry. C: Higher magnification of nNOS-immunopositive neurons reveals that the immunoprecipitate is restricted to the cytoplasm. D: Similar neuron population appears after NADPH-diaphorase enzyme histochemistry. Scale bars: A, B: $70 \mathrm{~mm}$; C: $30 \mathrm{~mm}$; D: $20 \mathrm{~mm}$ 


\section{Peptidergic innervation of nNOS-immunopositive neurons in the MS}

Since the examined area is rich in peptidergic axons, we carried out double labeling for $\mathrm{nNOS}$ and neuropeptides in order to reveal possible synaptic contacts between the two neurochemically different neuronal elements. Two neuropeptides, galanin (Fig. 2A) and leucine-enkephalin (Fig. 2B) were found to establish multiple close appositions (suggesting possible synaptic contacts) around nNOS-immunopositive cell bodies and proximal dendrites in the medial septum.
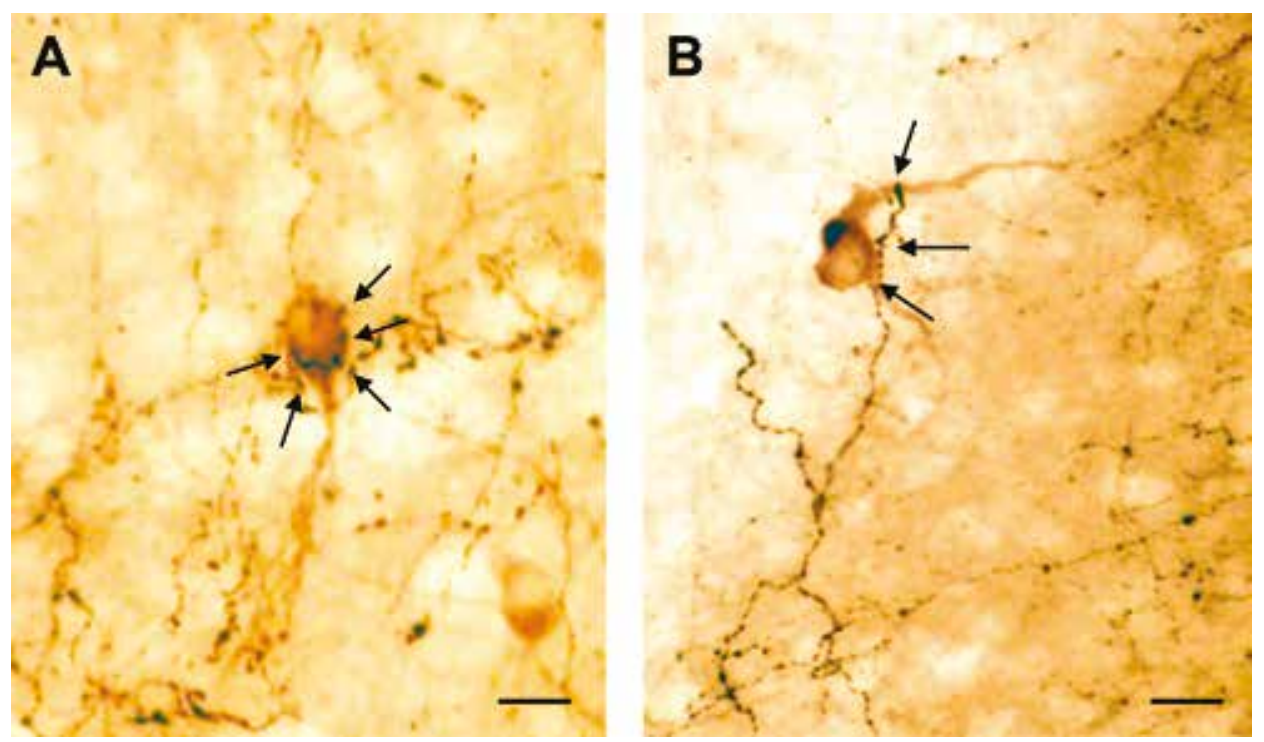

Fig. 2. Innervation of nNOS-immunopositive neurons by peptidergic axons in the MS. A: Varicose axons containing the neuropeptide galanin (black precipitate) form pericellular basket-like multiple contacts (arrows) around an nNOS-immunopositive cell body and proximal dendrites (brown precipitate). B: Multiple contacts (arrows) between a leu-enk-immunopositive varicose fiber (black precipitate) and an nNOS-immunopositive neuron (brown precipitate). Scale bar: $20 \mathrm{~mm}$

\section{Fine structure of nNOS-immunopositive neurons and their axons}

The nitrergic neurons of the medial septal area have large indented nuclei surrounded by a thin cytoplasmic rim (Fig. 3A). Numerous nNOS-positive axon terminals establishing both asymmetrical (Fig. 3B-D) and symmetrical (Fig. 4) synapses were identified. The targets of these synapses were mainly immunonegative dendritic shafts of various caliber (Fig. 3B) and rarely dendritic spines (Fig. 3D). nNOS-nNOS connections were also revealed (Fig. 3C, Fig. 4A, C) and the synapses between them were either asymmetrical (Fig. 3C) or symmetrical (Fig. 4).

The postembedding GABA-immunogold reaction revealed that the nNOS-terminals making symmetrical synapses also contain the inhibitory neurotransmitter, 

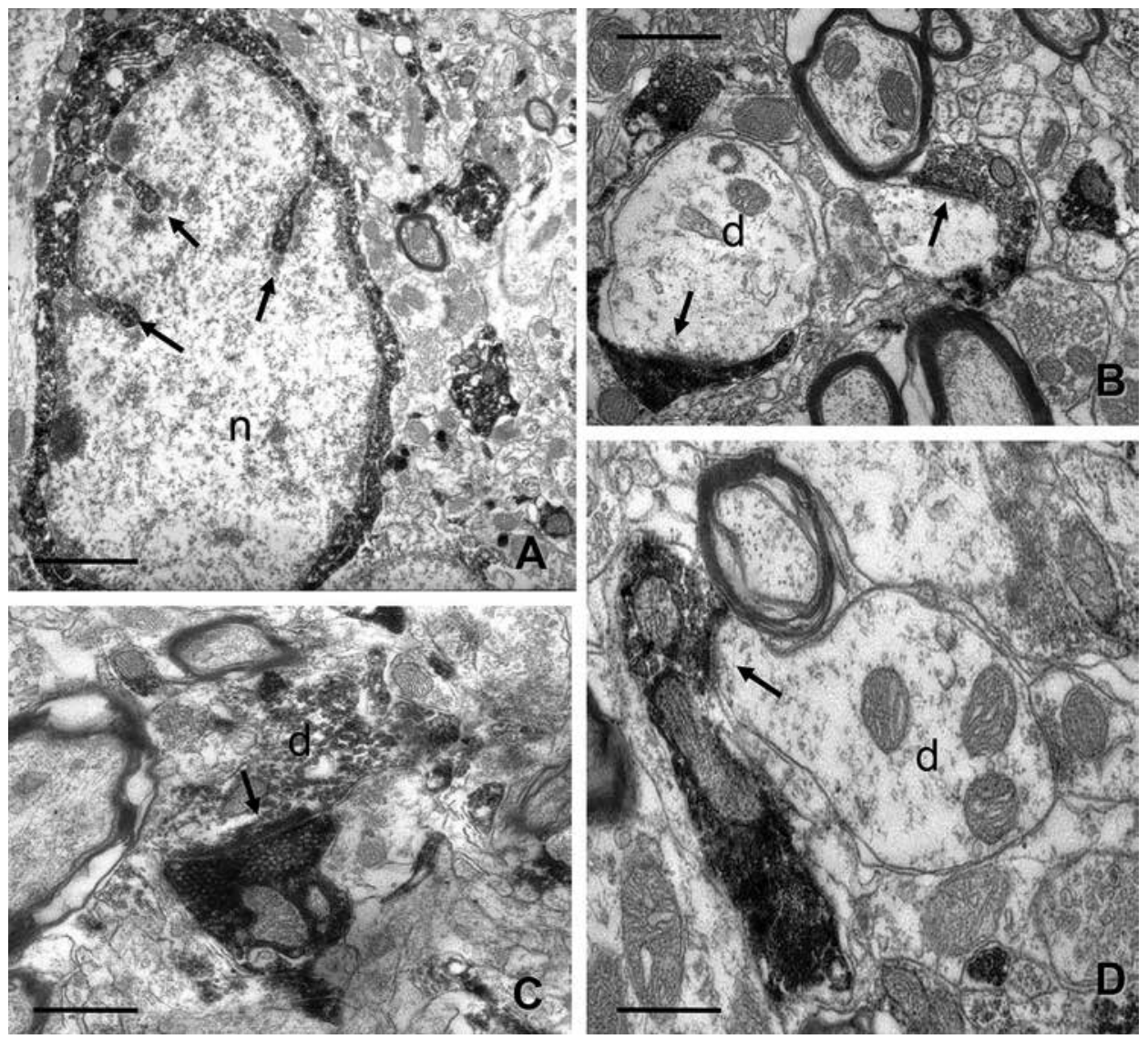

Fig. 3. Electron micrographs showing the fine structure and synaptology of nNOS-immunoreactive neuronal elements. A: A small ovoid neuronal soma with large, indented (arrows) nucleus (n). The cytoplasm is filled with dense immunoprecipitate. B: Asymmetrical synapses (arrows) of nNOS-immunopositive axon terminals with immunonegative dendritic shafts of varied caliber. C: Asymmetrical nNOS-nNOS connection (arrow) between an axon terminal and a dendritic shaft (d) D: Asymmetrical synapse (arrow) on a possible origin of dendritic spine. Scale bars: A: $1000 \mathrm{~nm}$; B, C, D: $250 \mathrm{~nm}$

GABA (Fig. 4A-C). Most of the synaptic boutons in asymmetrical synaptic contact were large and they contained round small agranular vesicles and several mitochondria (Fig. 3C, D), whereas the GABA-positive terminals usually contained ovoid small agranular vesicles and in most cases only one mitochondrion (Fig. 4) in the plane of the section. All nNOS-positive presynaptic elements in symmetric contacts were also labeled for GABA (Fig. 4A-C) and none of the asymmetric synapses were labeled.

Our semiquantitative target analysis (Fig. 5) including over 100 synaptic boutons $(n=113)$ revealed that $76 \%$ of the nitrergic terminals formed asymmetrical, and $24 \%$ 

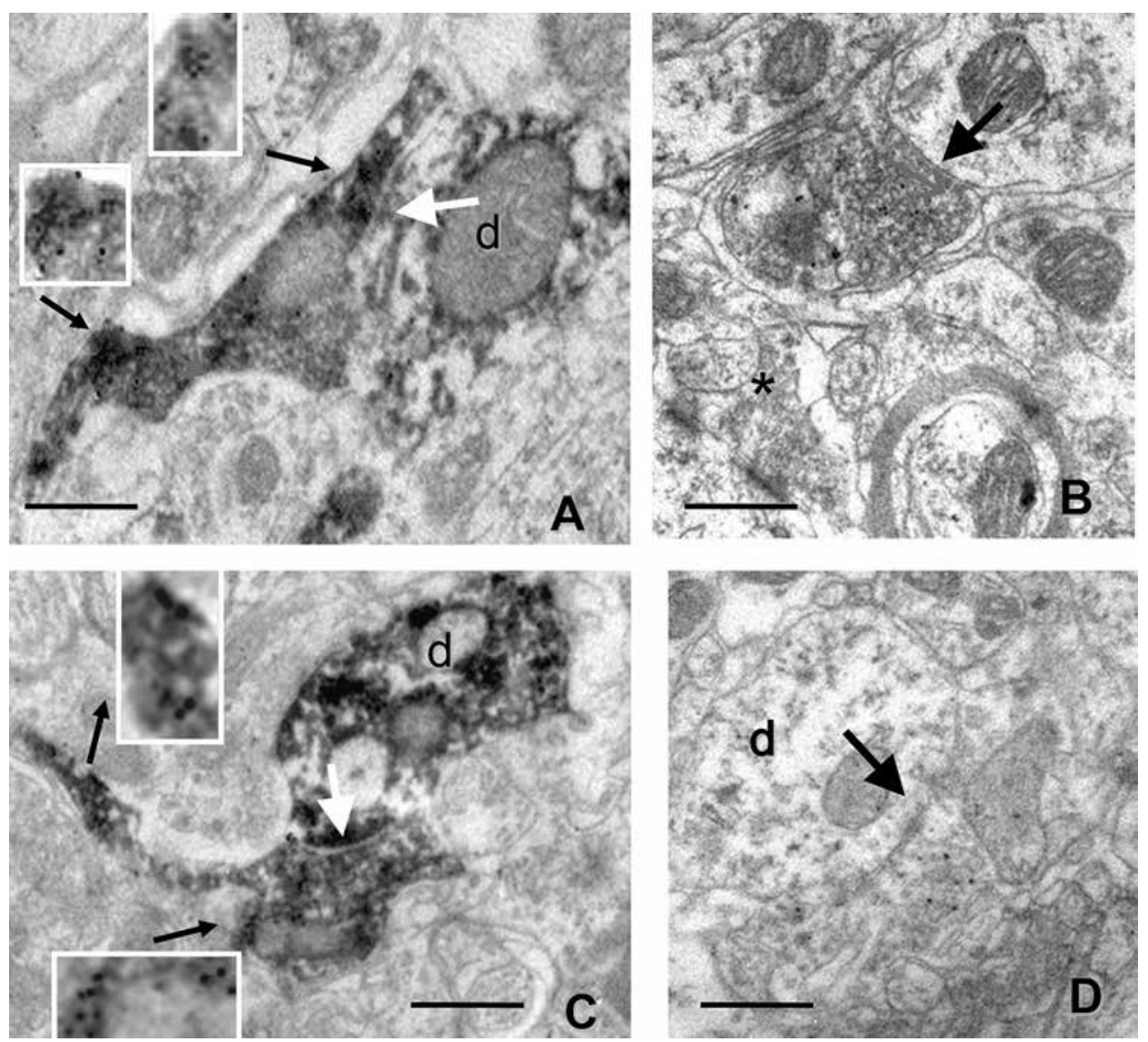

Fig. 4. Coexistence and connections of nNOS- and GABA-immunopositive neuronal elements: A, C: Symmetrical synapses (large arrow) of axon terminals double-labelled for nNOS (precipitate) and GABA (enrichment of gold particles, also shown by the inserts with higher magnification) on nNOS-positive dendritic shafts (d). B: Symmetrical synapse (arrow) of an axon terminal double-labelled for nNOS (precipitate) and GABA (gold particles) on an immunonegative dendritic shaft. D: Symmetrical synapse (arrow) of an axon terminal unlabelled for nNOS and labelled for GABA (gold particles) on an immunonegative dendritic shaft. Scale bar: $250 \mathrm{~nm}$; Inserts: $150 \mathrm{~nm}$

symmetrical synapses. A $80 \%$ of the postsynaptic targets were nNOS-immunonegative, and $19 \%$ were nNOS-immunopositive.

In the group of nNOS-immunonegative targets $61 \%$ of the total number of synapses were asymmetrical and only 19\% symmetrical synapses. Within the group of nNOS-immunoreactive targets the proportion of asymmetrical synapses was $15 \%$, and only $4 \%$ received symmetrical inputs. The dendritic spines were mainly immunonegative $(11 \%)$ only a few of them were immunopositive $(2 \%)$, and they received exclusively asymmetrical synapses from the nitrergic terminals. The amount of unidentifiable connections was $1 \%$. 


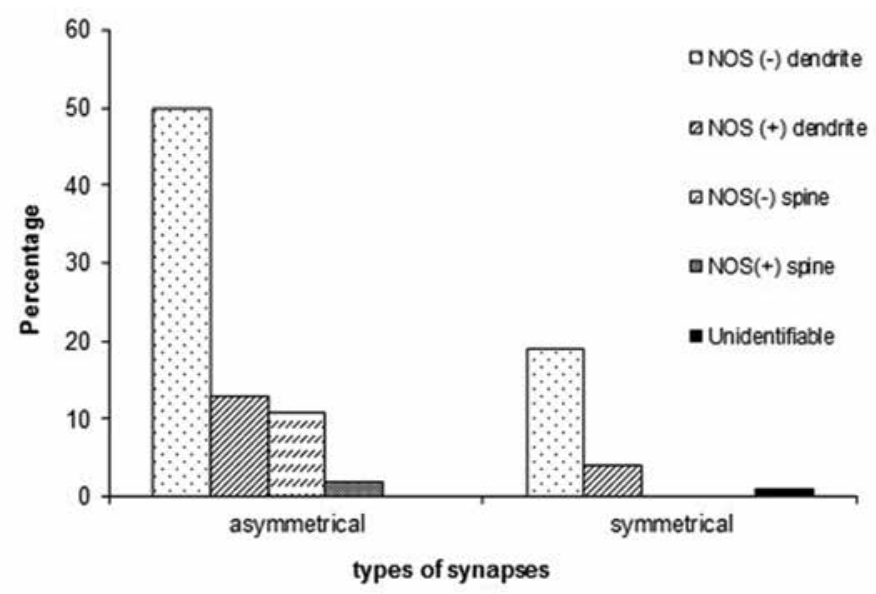

Fig. 5. Graph showing the percentage distribution of synaptic targets of nNOS-immunopositive axon terminals. The horizontal axis shows the type of the synapse, the vertical axis shows the occurrence of the type of postsynaptic targets in percentage of the total synapses $(n=113)$

\section{DISCUSSION}

The results show that the medial septal area of the rat is rich in nitrergic neuronal elements of both intrinsic and extrinsic origin. This is in contrast with the human septum, where the medial part has much less immunopositive cells and these are present more densely in the diagonal band of Broca [8]. We presented not only light microscopic, but also fine structural details and synaptology of nNOS-immunopositive septal neuronal elements. Fine structural immunocytochemical studies are rather rare in this field and they deal with other parts of the CNS, such as the spinal cord [43], nucleus of the solitary tract [5], periaqueductal grey matter [7], hypothalamus [1] or visual cortex [29]. Independently of the examined brain area the fine structural characteristics of the nNOS-immunopositive neurons, terminals and dendrites are astonishingly similar to each other as well as to our findings. In addition to neuronal presence Lüth [29] also detected weak astroglial labeling in the visual cortex. We could not visualize nNOS labeling in the glial cells of the medial septum, however, in our earlier studies we could detect VIP in astroglial processes in the interpeduncular nucleus [31].

Our studies confirm previous observations that nNOS-labeled and NADPHdiaphorase stained neurons are codistributed, and both methods are applicable for demonstrating the nitrergic system [13, 28]. The morphological characteristics of nitrergic neurons in the MS (large indented nucleus and narrow cytoplasmic rim) and the rarity of synaptic contacts on their somata suggest that they should be cholinergic neurons. Bialowas and Frotsher [10] have shown that ChAT-immunoreactive neurons possess large nuclei with multiple invaginations of the nuclear membrane and terminals rarely were found in synaptic contact on the cell bodies of positive neurons. This 
is further supported by the result of Kitchener and Diamond [26]: they had shown that $97 \%$ of NADPH-diaphorase reactive neurons in the MS/DB were choline acetyltransferase immunoreactive.

From among the various neuropeptides present in the MS we found possible inputs from galanin- and leucine enkephalin-containing axons to nNOS-containing somata. Since our previous studies failed to reveal galaninergic cell bodies in the MS, they may come from an extrinsic source. The presence of opioids in the septal area is well demonstrated [40] but their connection with the nitrergic cells has not been proven yet. In other areas the interaction between $\mathrm{NO}$ and opioid systems was demonstrated with the help of opioid receptor knockout mice [42, 45]. Opioids were shown to decrease inhibitory synaptic currents in cholinergic and GABAergic neurons. The close connection of opioidergic axons on the nNOS-immunopositive neurons provides morphological basis to the above electrophysiological findings.

We have presented for the first time a semiquantitative analysis at fine structural level about the synaptic contacts of nitrergic axon terminals and boutons based on their postsynaptic targets. A $19 \%$ of these targets seemed to be nNOS-positive, they are probably dendritic processes from the local cholinergic neurones. Because most of the nNOS/nNOS contacts were asymmetrical synapses, we presume the existence of an intrinsic excitatory circuit between nitrergic/cholinergic neurones, with a yet unknown functional role. In addition, most of the postsynaptic elements were nonnitrergic, their neurochemical characteristics have not been revealed in this study.

Several nitrergic contacts were symmetrical synapses $(24 \%)$, and we have confirmed that those nNOS-immunopositive terminals also contained GABA. nNOS was found to co-localize with GABA in several other areas of CNS [37, 43, 44]. Since in the MS there is no evidence for the existence of such (nNOS-GABA) co-localization, they may derive from an external source. Jinno and Kosaka [24] excluded the nNOS immunoreactivity of hippocampo-septal GABAergic projection. Another candidate may be the periaqueductal gray matter, where Barbaresi et al. [7] found several neurons with nNOS - calbindin co-localization. However, further investigation is needed to detect the origin of those neuronal processes.

In conclusion, here we presented data about the fine structure and synaptology of the nitrergic neuron population of the rat septum. We demonstrated overlap between nNOS-immunopositive and NADPH-diaphorase-positive elements, moreover galaninergic and opioidergic inputs to the nitrergic neurons. We established that these have ultrastructural features very similar to the findings in other brain areas. We revealed the postsynaptic target distribution of nitrergic neurons in the medial septum which may serve to the better understanding of the local circuits.

\section{ACKNOWLEDGEMENT}

This publication was supported by a Grant of the Hungarian Ministry of Human Resources, No. 114754/2016/FEKUT. 


\section{REFERENCES}

1. Affleck, V. S., Coote, J. H., Pyner, S. (2012) The projection and synaptic organization of NTS afferent connections with presympathetic neurons, GABA and nNOS neurons in the paraventricular nucleus of the hypothalamus. Neuroscience 219, 48-61.

2. Alonso, A., Köhler, C. (1984) A study of the reciprocal connections between the septum and the entorhinal area using anterograde and retrograde axonal transport methods in the rat brain. J. Comp. Neurol. 225, 327-343.

3. Amaral, D. G., Kurz, J. (1985) An analysis of the origins of the cholinergic and non-cholinergic septal projections to the hippocampal formation of the rat. J. Comp. Neurol. 240, 37-59.

4. Arendt, T., Bruckner, M. K., Bigl, V., Marcova, L. (1995) Dendritic reorganisation in the basal forebrain under degenerative conditions and its defects in Alzheimer's disease. III. The basal forebrain compared with other subcortical areas, J. Comp. Neurol. 351, 223-246.

5. Atkinson, L., Batten, T. F. C., Corbett, K. A., Sinfield, J. K., Deuchars, J. (2003) Subcellular localization of neuronal nitric oxide synthase in the rat nucleus of the solitary tract in relation to vagal afferent inputs. Neuroscience 118, 115-122.

6. Baisden, R. H., Woodruff, M. L., Hoover, D. B. (1984) Cholinergic and non-cholinergic septo-hippocampal projections: a double-label horseradish peroxidase-acetylcholinesterase study in the rabbit. Brain Res. 290, 146-151.

7. Barbaresi, P., Mensa, E., Lariccia, V., Pugnaloni, A., Amoroso, S., Fabri, M. (2013) Differential distribution of parvalbumin- and calbindin-D28K-immunoreactive neurons in the rat periaqueductal gray matter and their colocalization with enzymes producing nitric oxide. Brain Res. Bull. 99, 48-62.

8. Bartus, R. T. Dean, R. L., Beer, III, B., Lippa, A. S. (1982) The cholinergic hypothesis of geriatric memory dysfunction. Science 217, 408-414.

9. Bernstein, H.-G., Brisch, R., Ogonlade, V., Heinemann, A., Baumann, B., Arendt, T., Dobrowolny, H., Bogerts, B., Lüth, H.-J. (2004) Detection of nitric oxide synthase (NOS) immunoreactive neurons in the human septal area: a matter of method? J. Chem. Neuroanat. 27, 247-250.

10. Bialowas, J., Frotsher, M. (1987) Choline acetyltransferase-immunoreactive neurons and terminals in the rat septal complex: combined light and electron microscopic study. J. Comp. Neurol. 259, 298307.

11. Bland, B. H., Oddie, S. D. (1998) Anatomical, electrophysiological and pharmacological studies of ascending brain stem hippocampal synchronizing pathways, Neurosci. Biobehav. Rev. 22, 259-273.

12. Brashear, H. R., Zaborszky, L., Heimer, L. (1986) Distribution of GABAergic and cholinergic neurons in the rat diagonal band. Neuroscience 17, 439-451.

13. Bredt, D. S., Glatt, C. E., Hwang, P. M., Fotuhi, M., Dawson, T. M., Snyder, S. H. (1991) Nitric oxide synthase protein and mRNA are discreetly localized in neuronal populations of mammalian central nervous system together with NADPH diaphorase. Neuron 7, 615-624.

14. Bredt, D. S., Snyder, S. H. (1994) Nitric oxide: a physiologic messenger molecule. Annu. Rev Biochem. 63, 175-195.

15. Cheng, A., Wang, S., Cai, J., Rao, M. S., Mattson, M. P. (2003) Nitric oxide acts in a positive feedback loop with BDNF to regulate neural progenitor cell proliferation and differentiation in the mammalian brain. Dev. Biol. 258, 319-333.

16. Colas, D., Gharib, A., Bezin, L., Morales, A., Guidon, G., Cespuglio, R., Sarda, N. (2006) Regional age-related changes in neuronal nitric oxide synthase (nNOS), messenger RNA levels and activity in SAMP8 brain. BMC Neuroscience 20067:81. DOI: 10.1186/1471-2202-7-81.

17. Dinerman, J. L., Dawson, T. M., Schell, M. J., Snowman, A., Snyder, S. H. (1994) Endothelial nitric oxide synthase localized to hippocampal pyramidal cells: implications for synaptic plasticity. Proc. Natl Acad. Sci. USA 91, 4214-4218.

18. Getting, S. J., Segieth, J., Ahmad, S., Biggs, C. S., Whitton, P. S. (1996) Biphasic modulation of GABA release by nitric oxide in the hippocampus of freely moving rats in vivo. Brain Res. 717, 196-199. 
19. Guix, F. X., Uribesalgo, I., Coma, M., Munoz, F. J. (2005) The physiology and pathophysiology of nitric oxide in the brain. Prog. Neurobiol. 76, 126-152.

20. Hensley, K., Maidt, M. L., Yu, Z., Sang, H., Markesbery, W. R., Floyd, R. A. (1998) Electrochemical analysis of protein nitrotyrosine and dityrosine in the Alzheimer brain indicates region-specific accumulation. J. Neurosci. 18, 8126-8132.

21. Hodgson, A. J., Penke, B., Erdei, A., Chubb, I. W., Somogyi, P. (1985) Antiserum to gamma-aminobutyric acid. I. Production and characterization using a new model system. J. Histochem. Cytochem. $33,229-239$.

22. Holttum, J. R., Gershon, S. (1992) The cholinergic model of dementia, Alzheimer type. Progression from the unitary transmitter concept. Dementia 3, 174-185.

23. Jakab, R. L., Leranth, Cs. (1995) Septum. In: Paxinos, G. (ed.) The Rat Nervous system. Academic Press, London, pp. 405-441.

24. Jinno, S., Kosaka, T. (2002) Immunocytochemical characterization of hippocamposeptal projecting GABAergic nonprincipal neurons in the mouse brain: a retrograde labeling study. Brain Res. 945, 219-231.

25. Kiss, J., Patel, A. J., Freund, T. F. (1990) Distribution of septohippocampal neurons containing parvalbumin or choline acetyltransferase in the rat brain. J. Comp. Neurol. 298, 362-372.

26. Kitchener, P. D., Diamond, J. (1993) Distribution and colocalization of choline acetyltransferase immunoreactivity and NADPH diaphorase reactivity in neurons within the medial septum and diagonal band of Broca in the rat basal forebrain. J. Comp. Neurol. 335, 1-15.

27. Köhler, C., Chan-Palay, V. (1983) Distribution of gamma-aminobutyric acid containing neurons and terminals in the septal area. Anat. Embryol. 167, 53-65.

28. Laing, I., Todd, A. J., Heizmann, C. W., Schmidt, H. H. H. W. (1994) Subpopulations of GABAergic neurons in laminae I-III of rat spinal dorsal horn defined by coexistence with classical transmitters, peptides, nitric oxide synthase or parvalbumin. Neuroscience 61, 123-132.

29. Lüth, H.-J. (1997) Ultrastructural demonstration of constitutive nitric oxide synthase (cNOS) in neocortical glial cells and glial perisynaptic sheaths. Ann. Anat. 179, 221-225.

30. Monti, J. M., Jantos, H. (2004) Effects of the 5-HT1A receptor ligands flesinoxan and WAY 100635 given systemically or microinjected into the laterodorsal tegmental nucleus on REM sleep in the rat. Behav. Brain Res. 151, 159-166.

31. Paspalas, C., Halasy, K., Gerics, B., Papadopoulo, G., Hajós, F. (2001) Vasoactive intestinal polypeptide in neuroglia? Immunoelectron microscopic localization in astrocytes of the rat mesencephalon. Glia 34, 229-233.

32. Pasqualotto, B. A., Vincent, S. R. (1991) Galanin and NADPH-diaphorase coexistence in cholinergic neurons of the rat basal forebrain. Brain Res. 551, 78-86.

33. Prast, H., Tran, M. H., Fischer, H., Philippu, A. (1998) Nitric oxide-induced release of acetylcholine in the nucleus accumbens: role of cyclic GMP, glutamate and GABA. J. Neurochem. 71, 266-273.

34. Prast, H., Philippu, A. (2001) Nitric oxide as modulator of neuronal function. Prog. Neurob. 64, 51-68.

35. Rodrigo, J., Springall, D. R., Uttenthal, O., Bentura, M. L., Abadia-Molina, F., Riveros-Moreno, V., Martinez-Murillo, R., Polak, J. M., Moncada, S. (1994) Localization of nitric oxide synthase in the adult rat brain. Philos. Trans R Soc. Lond B Biol. Sci. 345, 175-221.

36. Rye, D. B., Wainer, B. H., Mesulam, M.-M., Mufson, E. J., Saper, C. B. (1984) Cortical projections arising from the basal forebrain: a study of cholinergic and non-cholinergic components employing combined retrograde tracing and immunohistochemical localization of choline acetyltransferase. Neuroscience 13, 627-643.

37. Sardella, T. C. P., Polgár, E., Watanabe, M., Todd, J. (2011) A quantitative study of neuronal nitric oxide synthase expression in laminae I-III of the rat spinal dorsal horn. Neuroscience 192, 708-720.

38. Sequeira, S. M., Ambrósio, A. F., Malva, J. O., Carvalho, A. P., Carvalho, C. M. (1997) Modulation of glutamate release from rat hippocampal synaptosomes by nitric oxide. Nitric Oxide 1, 315-329. 
39. Sotty, F., Danik, M., Manseau, F., Laplante, F., Quirion, R., Williams, L. (2003) Distinct electrophysiological properties of glutamatergic, cholinergic and GABAergic rat septohippocampal neurons: novel implications for hippocampal rhythmicity, J. Physiol. (Lond.) 551, 927-943.

40. Szeidemann, Z., Shanabrough, M., Leranth, C. (1995) Hypothalamic Leu-enkephalin-immunoreactive fibers terminate on calbindin-containing somatospiny cells in the septal area of the rat. J. Comp. Neurol. 358, 573-583.

41. Tran, M. H., Yamada, K., Nakajima, A., Mizuno, M., He, J., Kamei, H., Nabeshima, T. (2003) Tyrosine nitration of a synaptic protein synaptophysin contributes to amyloid beta-peptide-induced cholinergic dysfunction. Mol. Psychiatry. 8, 407-412.

42. Yoo, J. H., Cho, J. H., Lee, S. Y., Loh, H. H., Ho, I. K., Jang, C. G. (2005) Reduced nNOS expression induced by repeated nicotine treatment in $\mu$-opioid receptor knockout mice. Neurosci. Letters 380 , 70-74.

43. Waltschanoff, J., Weinberg, R. J., Rustioni, A., Schmidt, H. H. H. W. (1992) Nitric oxide synthase and GABA colocalize in lamina II of spinal cord. Neurosci. Letters 148, 6-10.

44. Waltschanoff, J., Weinberg, R. J., Kharazia, V. N., Schmidt, H. H. H. W., Nakane, M., Rustioni, A (1993) Neurons in rat cerebral cortex that synthesize nitroc oxide: NADPH diaphorase histochemistry, NOS immunocytochemistry and colocalization with GABA. Neurosci. Letters 157, 157-161.

45. Zochone, D. W., Hong Sun, Xia-Qing Li (2001) Evidence that nitric oxide- and opioid-containing interneurons innervate vessels in the dorsal horn of the spinal cord of rats. J. Physiol. 532.3, 749-758. 